\title{
Dynamics of Anomalous Stratospheric Eddy Heat Flux Events in an Idealized Model $^{\mathscr{O}}$
}

\author{
ETIENNE DUNN-SIGOUIN \\ Geophysical Institute, University of Bergen, and Bjerknes Centre for Climate Research, Bergen, Norway \\ TIFFANY SHAW \\ Department of the Geophysical Sciences, University of Chicago, Chicago, Illinois
}

(Manuscript received 27 August 2019, in final form 3 April 2020)

\begin{abstract}
Extreme stratospheric eddy and sudden stratospheric warming (SSW) events both involve anomalous stratospheric eddy heat flux. The cause of the anomaly has been hypothesized to be due to tropospheric or stratospheric dynamics. Here, ensemble spectral nudging experiments in a dry dynamical-core model are used to quantify the role of the troposphere versus the stratosphere. The experiments focus on the wavenumber- 1 heat flux since it dominates the anomalous stratospheric eddy heat flux during both events. Nudging the stratospheric zonal-mean flow does not account for the anomalous stratospheric wave- 1 heat flux. Nudging either tropospheric wave-1 or higher-order wavenumbers $(k \geq 2)$ accounts for a large fraction of the anomalous stratospheric wave-1 heat flux. Mechanism denial experiments, whereby tropospheric eddies (wave 1 or $k \geq 2$ ) are nudged and the zonal-mean flow is fixed to climatology, suggest the climatological stratospheric zonal-mean flow is sufficient to account for the anomalous stratospheric wave-1 heat flux and wave-wave interaction plays a role in generating the anomalous tropospheric wave- 1 source. Taken together, the experiments suggest the troposphere dominates the anomalous stratospheric eddy heat flux during extreme stratospheric eddy and SSW events while the stratospheric zonal-mean flow plays secondary role.
\end{abstract}

\section{Introduction}

Vertical coupling by planetary-scale waves dominates the variability of the wintertime stratospheric circulation (Plumb 2010). Upward planetary wave coupling from the troposphere to the stratosphere is often diagnosed using the zonal-mean meridional eddy heat flux $\overline{v^{\prime} T^{\prime}}$, where primes denote deviations from the zonal mean (eddies) and the bar denotes the zonal mean, since positive values are consistent with an upward wave group velocity according to linear theory (Andrews et al. 1987). Diagnostic studies based on reanalysis data show that 1) cumulative anomalous stratospheric eddy flux occurs prior to sudden stratospheric warming (SSW) events (e.g., Limpasuvan et al. 2004; Polvani and Waugh

\footnotetext{
Supplemental information related to this paper is available at the Journals Online website: https://doi.org/10.1175/JAS-D-190231.s1.
}

\footnotetext{
Corresponding author: Etienne Dunn-Sigouin, etienne.dunn-sigouin@ uib.no
}

2004; Dunn-Sigouin and Shaw 2015), commonly defined by the reversal of the zonal-mean zonal wind from westerly to easterly at $10 \mathrm{hPa}$ and $60^{\circ} \mathrm{N}$ (Charlton and Polvani 2007) and 2) extreme instantaneous stratospheric eddy heat flux events, defined by the 95th percentile of the daily 50 -hPa wave- 1 heat flux in highlatitudes $\left(60^{\circ}-90^{\circ} \mathrm{N}\right)$ (Dunn-Sigouin and Shaw 2015), occur prior to zonal-mean flow deceleration events (Martineau and Son 2015; Birner and Albers 2017; de la Cámara et al. 2019). Previous work by Dunn-Sigouin and Shaw (2015) showed SSWs and extreme stratospheric eddy (deceleration) events are largely distinct events in reanalysis data.

Extreme stratospheric eddy and SSW events both involve anomalous stratospheric eddy heat flux; however, the cause of the anomaly is not clear. It has been hypothesized to be due to tropospheric or stratospheric dynamics. The troposphere dominates view assumes an anomalous tropospheric source of wave activity that propagates upward into the stratosphere when the stratospheric zonal-mean flow is westerly (e.g., Charney and Drazin 1961; Matsuno 1970, 1971; Karoly and Hoskins 
1982). Thus, an anomalous tropospheric wave source is critical whereas a specific configuration of the stratospheric zonal-mean flow is not. Harnik (2009) and Sjoberg and Birner (2012) suggest the time scale of the anomalous tropospheric wave source is key.

In contrast, the stratosphere dominates view assumes a preconditioned stratospheric zonal-mean flow involving a strengthened vortex that 1 ) focuses wave activity upward into the stratosphere (e.g., Holton and Mass 1976; McIntyre 1982; Chen and Robinson 1992; Scott and Polvani 2006; Sjoberg and Birner 2014; Birner and Albers 2017) or 2) forms a two- or three-sided cavity favoring resonant wave amplification (e.g., Clark 1974; Tung and Lindzen 1979; Plumb 1981, 2010; Matthewman and Esler 2011; Esler and Matthewman 2011). Thus, a sufficiently large climatological wave source in the troposphere is necessary; however, the wave source is not required to be anomalous. Instead, a specific configuration of the stratospheric zonal-mean flow is critical to produce the anomalous stratospheric heat flux.

Several previous studies have attempted to address the relative importance of the stratosphere versus the troposphere for planetary wave coupling. For example, model studies tested the role of the stratosphere or troposphere separately by modifying the boundary conditions or relaxing the circulation toward a specific evolution (Reichler et al. 2005; Hitchcock and Haynes 2016; de la Cámara et al. 2017; Martineau et al. 2018). However, the results are difficult to interpret because the role of the stratosphere and troposphere were not compared quantitatively. Some studies are diagnostic (e.g., Albers and Birner 2014; Jucker 2016; Birner and Albers 2017; Lindgren et al. 2018; White et al. 2019; Domeisen et al. 2018; de la Cámara et al. 2019) making it difficult to infer causality. Other studies used highly truncated models (e.g., Clark 1974; Holton and Mass 1976; Tung and Lindzen 1979; Plumb 1981; Chen and Robinson 1992; Scott and Polvani 2006; Esler and Matthewman 2011; Domeisen and Plumb 2012; Sjoberg and Birner 2014) making it difficult to assess whether the results carry over to the real atmosphere. The study by Sun et al. (2012) used a dry dynamical-core model to perturb stratospheric and tropospheric initial conditions preceding SSW events and found the troposphere is key to predict the events. However, initial-condition experiments do not directly show causality since they do not control the troposphere and stratosphere throughout the duration of the events and cannot compare the roles of the tropospheric wave source versus the stratospheric zonal-mean flow since all wavenumbers are perturbed.
Here, we focus on quantifying the role of the troposphere versus the stratosphere using ensemble spectral nudging experiments in an idealized dry dynamical-core model. We use spectral nudging to relax the components toward a prescribed evolution to determine their impact on the freely evolving circulation (e.g., Douville 2009; Greatbatch et al. 2012; Dunn-Sigouin and Shaw 2018). We perform experiments nudging the eddies and the zonal-mean flow separately and in combination to test which components can account for the anomalous stratospheric eddy heat flux during extreme stratospheric eddy heat flux and SSW events. The deterministic response is averaged over an ensemble initialized from a set of independent initial conditions. The nudging experiments are performed to answer the following questions:

1) Does the troposphere or stratosphere dominate the dynamics of extreme stratospheric eddy and SSW events?

If the troposphere dominates, then 1) nudging the tropospheric eddies alone should reproduce the anomalous stratospheric eddy heat flux whereas 2) nudging the stratospheric zonal-mean flow alone should not reproduce the anomalous stratospheric eddy heat flux. If the stratosphere dominates, then 1) nudging the stratospheric zonal-mean flow alone should reproduce the anomalous stratospheric eddy heat flux whereas 2) nudging the tropospheric eddies alone should not reproduce the anomalous stratospheric eddy heat flux.

2) Do wave-wave interactions play a role during extreme stratospheric eddy and SSW events?

The troposphere dominates view involves an anomalous tropospheric wave source while the stratosphere dominates view involves a preconditioned stratospheric zonal-mean flow but neither specifies their origin. Wave-wave interactions could generate the tropospheric wave source directly via interactions between wavenumbers (Scinocca and Haynes 1998; Schneidereit et al. 2017; Lee et al. 2019) or indirectly such that tropospheric waves modify the zonal-mean flow impinging on surface topography (Charney and Eliassen 1949). Wave-wave interactions could be mediated by the stratospheric zonal-mean flow such that stratospheric waves precondition the polar vortex for upward wave propagation or resonance (McIntyre 1982; Palmer and Hsu 1983; Albers and Birner 2014). Finally, wave-wave interaction could directly generate the stratospheric eddy heat flux independent of the troposphere (Smith 1983; Smith et al. 1984). Nudging specific wavenumbers should clarify if and how wave-wave interactions play a role. 
TABLE 1. Summary of the ensemble spectral nudging experiments as a function of event type, zonal wavenumber, vertical domain, and lag centered on each event. Experiments 1-14 are the main results while experiment A1 is a sensitivity experiment in the appendix. The stratospheric and tropospheric vertical domains are defined as hybrid-sigma levels above and below $137 \mathrm{hPa}$, respectively. An ensemble composed of 50 members is produced for each of the 15 extreme stratospheric eddy and SSW events from the model. The initial conditions are taken from the control simulation every 50 days. We note that some ensemble members in experiment 11 failed to integrate to completion. Therefore, here we use the successfully integrated ensemble members from experiment 11 performed using a shorter model time step and 100 ensemble members, which resulted in a failure rate of $30 \%$ for 2 events. Experiment A 1 controls the higher-order wavenumbers continuously toward the evolution of the control simulation from days 500 to 3500 in a single integration.

\begin{tabular}{|c|c|c|c|c|}
\hline Expt & Event type & Wavenumber & Vertical domain & Lag (days) \\
\hline 1 & Extreme stratospheric eddy & k1 & Troposphere & -20 to 20 \\
\hline 2 & SSW & k1 & Troposphere & -40 to 40 \\
\hline 3 & Extreme stratospheric eddy & k0 & Stratosphere & -20 to 20 \\
\hline 4 & SSW & k0 & Stratosphere & -40 to 40 \\
\hline \multirow[t]{2}{*}{5} & Extreme stratospheric eddy & k0 & Stratosphere & -20 to 20 \\
\hline & & $\mathrm{k} 1$ & Troposphere & -20 to 20 \\
\hline \multirow[t]{2}{*}{6} & SSW & k0 & Stratosphere & -40 to 40 \\
\hline & & $\mathrm{k} 1$ & Troposphere & -40 to 40 \\
\hline \multirow[t]{2}{*}{7} & Extreme stratospheric eddy & k0 (climatology) & Stratosphere & -20 to 20 \\
\hline & & $\mathrm{k} 1$ & Troposphere & -20 to 20 \\
\hline \multirow[t]{2}{*}{8} & SSW & k0 (climatology) & Stratosphere & -40 to 40 \\
\hline & & $\mathrm{k} 1$ & Troposphere & -40 to 40 \\
\hline 9 & Extreme stratospheric eddy & $k \geq 2$ & Troposphere & -20 to 20 \\
\hline 10 & SSW & $k \geq 2$ & Troposphere & -40 to 40 \\
\hline 11 & Extreme stratospheric eddy & $k \geq 2$ & Stratosphere & -20 to 20 \\
\hline 12 & SSW & $k \geq 2$ & Stratosphere & -40 to 40 \\
\hline \multirow[t]{2}{*}{13} & Extreme stratospheric eddy & k0 (climatology) & Troposphere & -20 to 20 \\
\hline & & $k \geq 2$ & Troposphere & -20 to 20 \\
\hline \multirow[t]{2}{*}{14} & SSW & k0 (climatology) & Troposphere & -40 to 40 \\
\hline & & $k \geq 2$ & Troposphere & -40 to 40 \\
\hline A1 & - & $k \geq 2$ & Troposphere & 500 to 3500 \\
\hline
\end{tabular}

The paper is organized as follows. Section 2 presents the data, diagnostics and experiments used in this study. Section 3a compares the tropospheric and stratospheric components during extreme stratospheric eddy and SSW events in the model with those in reanalysis data. Section $3 b$ tests whether the troposphere or stratosphere dominates the dynamics of extreme stratospheric eddy and SSW events. Section $3 c$ quantifies the role of wavewave interaction during the events. Finally, the conclusions are summarized and discussed in section 4.

\section{Data, diagnostics, and experiments}

\section{a. Dry dynamical-core model and reanalysis data}

We integrate a freely evolving control simulation using the Geophysical Fluid Dynamics Laboratory's spectral dry dynamical-core model. The model integrates the primitive equations on a sphere and is driven by idealized physics: Newtonian relaxation of temperature toward a specified zonal-mean profile, Rayleigh friction near the surface, and a stratospheric sponge layer near the model lid. The control simulation is configured for perpetual-winter conditions using a horizontal resolution of triangular truncation 42 (T42) and a vertical resolution of 40 hybrid-sigma vertical levels. Stationary waves are generated in the model by setting the surface geopotential to a 4000-m Gaussian zonal wavenumber $\mathrm{k} 1$ spanning $25^{\circ}-65^{\circ} \mathrm{N}$ centered at $45^{\circ} \mathrm{N}$. The control simulation is run for 20000 days where the first 500 days of spin-up are discarded. A complete description of the control simulation is found in Table 1 and section 2 of Dunn-Sigouin and Shaw (2018). All data are linearly interpolated to pressure levels and daily data are used for all calculations. Meridional averages are cosine weighted and a 5-day running-mean smoothing is applied when plotting. Anomalies are defined as a deviation from the control simulation time-mean climatology.

We compare the control simulation to daily ERA-Interim data (Dee et al. 2011) during Northern Hemisphere (NH) December-March (DJFM) from 1979 to 2012. Anomalies are defined as a deviation from the daily-mean seasonal cycle.

\section{b. Diagnostics}

\section{1) EXTREME STRATOSPHERIC EDDY EVENTS}

Extreme stratospheric eddy events in reanalysis data are defined following Dunn-Sigouin and Shaw (2015) by extreme positive values of the daily January-March (JFM) zonal-mean wave-1 meridional heat flux $\left(\overline{v^{\prime} T_{k=1}^{\prime}}\right)$ averaged from $60^{\circ}$ to $90^{\circ} \mathrm{N}$ at $50 \mathrm{hPa}$. The high-latitude 
heat flux is used because stratospheric wave- 1 variability peaks in high latitudes (Shaw and Perlwitz 2013) and JFM corresponds to the period of maximum planetary wave coupling in the Northern Hemisphere (Shaw et al. 2010). Extreme stratospheric eddy events are identified when the 5-day running-mean high-latitude heat flux rises above the 95th percentile of the climatological distribution $\left(59.3 \mathrm{~K} \mathrm{~m} \mathrm{~s}^{-1}\right)$. The central date (day 0 ) of the events is defined at the day of maximum high-latitude heat flux and each event must be separated by a minimum of 15 days. The definition identifies 25 events in ERA-Interim from 1979 to 2012 and is relatively insensitive to the time scale of running-mean smoothing of the heat flux (Table 1 of Dunn-Sigouin and Shaw 2015).

Extreme stratospheric eddy events in the control simulation are defined using the 95th-percentile threshold in the control simulation $\left(63.7 \mathrm{~K} \mathrm{~m} \mathrm{~s}^{-1}\right)$. Due to computational and storage constraints for experiments discussed below, a subset of the strongest 15 events are used corresponding to the 99.5 th percentile $\left(90.4 \mathrm{~K} \mathrm{~m} \mathrm{~s}^{-1}\right)$. Key results (experiments 1,3 , and 9 in Table 1) are reproduced using the strongest 30 extreme stratospheric eddy events that exhibit similar composite life cycles.

\section{2) STRATOSPHERIC SUDDEN WARMING EVENTS}

SSW events in reanalysis data are defined following Charlton and Polvani (2007) when the daily DJFM zonal-mean zonal wind at $10 \mathrm{hPa}$ and $60^{\circ} \mathrm{N}$ first reverses from westerly to easterly. The zonal wind must return to westerly for 20 consecutive days between events and must return to westerly for at least 10 consecutive days following each event prior to 30 April. We note that this definition does not separate between different types of SSW events (e.g., split or displacement). The definition identifies 23 events in ERA-Interim from 1979 to 2012 (Table 2 in Butler et al. 2017). One-third of the SSW events in reanalysis data are preceded by extreme stratospheric eddy events within 30 days. Similar overlap is found for different time scales of runningmean smoothing of the heat flux used to define extreme stratospheric eddy events, confirming the events are largely distinct.

SSW events in the control simulation are defined as in reanalysis except for the 30 April criterion since the model is run in a perpetual-winter configuration. The control simulation produces an SSW frequency of 1 event every 197 days consistent with 1 event every other winter in reanalysis data. A subset of $15 \mathrm{SSW}$ events is selected based on the strongest cumulative 30-day highlatitude wave- 1 heat flux at $50 \mathrm{hPa}$ prior to the events motivated by previous work (Polvani and Waugh 2004; Dunn-Sigouin and Shaw 2015). We confirm that none of the $15 \mathrm{SSW}$ events in the control simulation are preceded by the 15 extreme stratospheric eddy events within 30 days. Key results (experiments 2, 4, and 10 in Table 1) are reproduced using the strongest $30 \mathrm{SSW}$ events based on the cumulative wave- 1 heat flux that exhibit similar composite life cycles.

\section{3) NORTHERN ANNULAR MODE}

The northern annular mode (NAM) is used to diagnose the strength of the zonal-mean flow during extreme stratospheric eddy and SSW events following previous work (e.g., Baldwin and Dunkerton 2001; Dunn-Sigouin and Shaw 2015). In the control simulation and reanalysis data, the NAM is defined as the standardized principle component time series of the first EOF of daily zonalmean geopotential height anomalies from $0^{\circ}$ to $90^{\circ} \mathrm{N}$ at each pressure level. The geopotential height anomalies are defined following Gerber et al. (2010). The global mean from each pressure level and day is removed at each latitude. Then, the daily mean seasonal cycle and the linear trend is removed from reanalysis data for each day of the year whereas the time-mean climatology is removed from the control simulation data. The resulting anomalies are weighted by the square root of the cosine of latitude. In the nudging experiments, the NAM is calculated by projecting the zonal-mean geopotential height anomalies on the leading EOF at each pressure level calculated from the control simulation.

\section{c. Nudging experiments}

We investigate the dynamical mechanisms of the anomalous stratospheric eddy heat flux during extreme stratospheric eddy and SSW events using spectral nudging following Dunn-Sigouin and Shaw (2018). Nudging uses Newtonian relaxation to constrain the tendencies of the spectral components $k$ of vorticity, divergence, temperature and surface pressure $X$ to a prescribed reference state $[\cdots]$ in the form $C(p)\left[X_{k}-\left(X_{k}\right)\right] / \tau$, where $\tau$ is a nudging time scale and $C(p)$ is a pressure dependent coefficient. Specifically, the zonal-mean flow $k=0$, wavenumber $k=1$, and higher-order wavenumbers $k \geq 2$ are nudged to the evolution of a given control simulation event. Nudging is performed on the native hybrid-sigma grid using a time scale of $\tau=1 / 10$ day. The response to the nudging is determined by averaging over an ensemble initialized from a set of 50 independent initial conditions taken every 50 days from the control run. Ensemble members for extreme stratospheric eddy events are integrated for 51 days from days -30 to +20 centered around the events. Similar but longer integrations are performed for SSW events (91 days from days -50 to +40$)$.

We isolate the role of stratospheric versus tropospheric dynamics by varying the nudging with height. 
The climatological tropopause in the control simulation varies from $137 \mathrm{hPa}$ in the tropics to $298 \mathrm{hPa}$ in the Arctic, where the pressure values are defined on hybridsigma levels assuming a constant surface pressure of $1000 \mathrm{hPa}$. Here we define stratospheric and tropospheric nudging above and below the tropical tropopause at $137 \mathrm{hPa}$; however, qualitatively similar results are found when using the Arctic tropopause at $298 \mathrm{hPa}$ except for a specific case discussed in section $3 b$. Specifically, stratospheric nudging varies $C(p)$ linearly from a value of 1 above $p_{t}=80 \mathrm{hPa}$ to 0 below $p_{b}=137 \mathrm{hPa}$ :

$$
C(p)=\left\{\begin{array}{lll}
1 & \text { if } \quad p<p_{t} \\
\frac{p-p_{b}}{p_{t}-p_{b}} & \text { if } \quad p_{t}<p<p_{b} . \\
0 & \text { if } \quad p>p_{b}
\end{array}\right.
$$

Tropospheric nudging varies linearly from 0 above $p_{t}=137 \mathrm{hPa}$ to 1 below $p_{b}=221 \mathrm{hPa}$ :

$$
C(p)=\left\{\begin{array}{lll}
0 & \text { if } \quad p<p_{t} \\
\frac{p-p_{t}}{p_{b}-p_{t}} & \text { if } \quad p_{t}<p<p_{b} . \\
1 & \text { if } \quad p>p_{b}
\end{array}\right.
$$

To determine robust mechanisms across individual events, we perform identical nudging for each of the 15 different extreme stratospheric eddy and SSW events from the control simulation for each experiment listed in Table 1. The response is shown as the composite of 15 ensemble-mean simulations for comparison with the composite of 15 control events. The robustness of the response across events is assessed by performing a twotailed Student's $t$ test using the spread of the 15 ensemblemean responses. Finally, we note that nudging a given zonal wavenumber in the sigma coordinate troposphere could inadvertently influence other wavenumbers in the pressure coordinate troposphere and stratosphere. We confirm this does not impact our results and the reader is referred to section 2d in Dunn-Sigouin and Shaw (2018) for further details.

\section{Results}

We begin by confirming the model captures the tropospheric and stratospheric components of extreme stratospheric eddy and SSW events in reanalysis data. Next, we present nudging experiments that test the troposphere and stratosphere dominates views. Finally, we present experiments that nudge specific wavenumbers in order to quantify the role of wave-wave interaction.

\section{a. Comparing extreme stratospheric eddy and SSW events in the model with reanalysis}

During extreme stratospheric eddy events, the wave-1 component accounts for the instantaneous (days -3 to +3 ) anomalous eddy heat flux in both reanalysis and the model (Figs. 1a,b). This is unsurprising given the wave-1 definition of extreme stratospheric eddy events. However, the wave- 1 component also accounts for the cumulative (days -30 to 0 ) anomalous eddy heat flux during the majority of SSW events in both reanalysis and the model (Figs. 1c,d). There are some notable exceptions in reanalysis data, for example, the split SSW events of January 1985, February 1989, and January 2009 (e.g., Charlton and Polvani 2007; Butler et al. 2017), which are dominated by higher-order wavenumbers (unfilled blue circles in Figs. 1c,d). Qualitatively similar results are found for different meridional and time averaging (e.g., $45^{\circ}-75^{\circ} \mathrm{N}$ and days -10 to 0 ) or pressure level (e.g., 30 or $70 \mathrm{hPa}$ ). Thus, the wave- $1 \mathrm{com}$ ponent dominates the anomalous stratospheric eddy heat flux during the events in both reanalysis and the model. Therefore, from this point onward we focus on the wave-1 heat flux.

Extreme stratospheric eddy events in reanalysis show an instantaneous positive stratospheric wave-1 heat flux anomaly around day 0 , which is qualitatively captured by the model but occurs lower down (Figs. 2a,b). In comparison, SSW events in reanalysis show a cumulative positive stratospheric wave- 1 heat flux anomaly from days -30 to 0 , which is also qualitatively captured by the model but occurs lower down (Figs. 2c,d). We note that in both reanalysis and the model, the magnitude of the anomalous stratospheric wave-1 heat flux during extreme stratospheric eddy events crosses the 95th-percentile threshold whereas it does not prior to SSW events, showing that SSWs are not extreme with respect to the wave-1 heat flux. Overall, the model qualitatively captures the anomalous stratospheric wave-1 heat flux during reanalysis events.

Extreme stratospheric eddy events in reanalysis show a positive tropospheric wave- 1 heat flux anomaly at $40^{\circ}-70^{\circ} \mathrm{N}$ from days -10 to 0 , which is qualitatively captured by the model (Figs. 3a,b). A similar positive tropospheric wave-1 heat flux anomaly occurs prior to SSW events in reanalysis from days -20 to 0 , which is also qualitatively captured by the model (Figs. 3c,d). The $40^{\circ}-70^{\circ} \mathrm{N}$ band is chosen because it captures the largest tropospheric wave- 1 heat flux anomalies prior to events in reanalysis and the model. We note that in all cases the anomalous lower-tropospheric wave- 1 heat flux is amplified but not extreme; that is, it does not cross the 95th percentile. Thus, the model qualitatively captures 


\section{extreme stratospheric eddy}

(a)

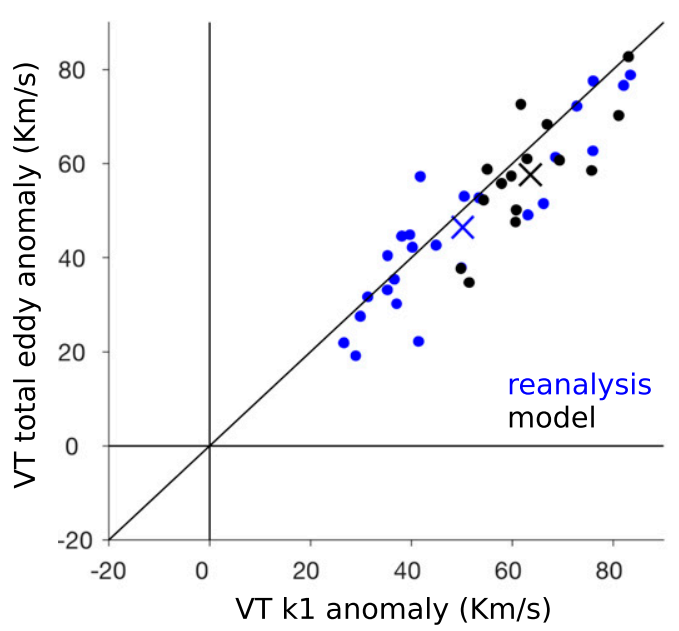

(b)

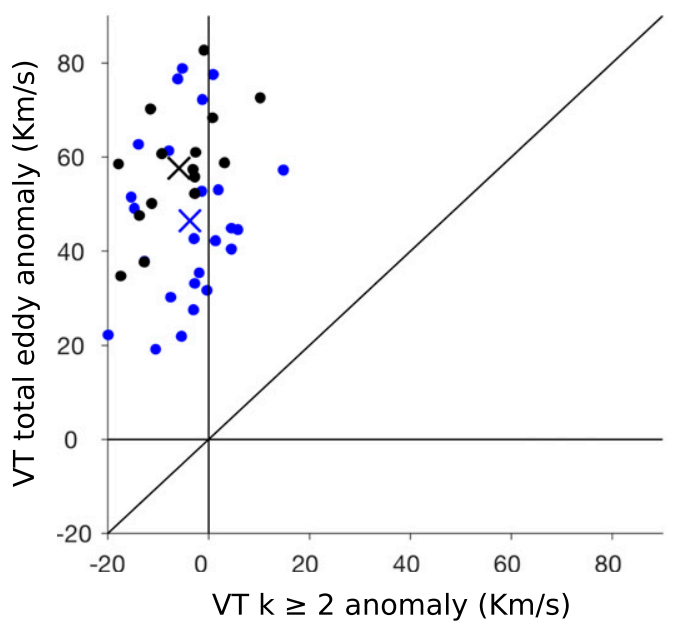

SSW

(c)

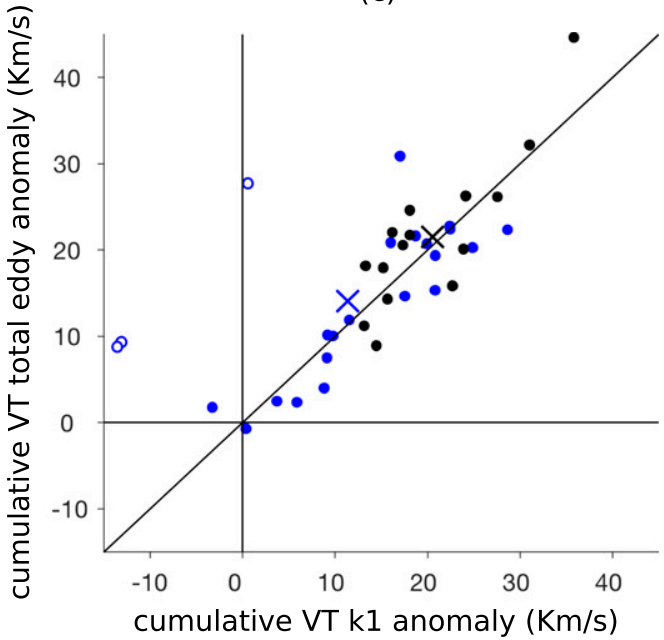

(d)

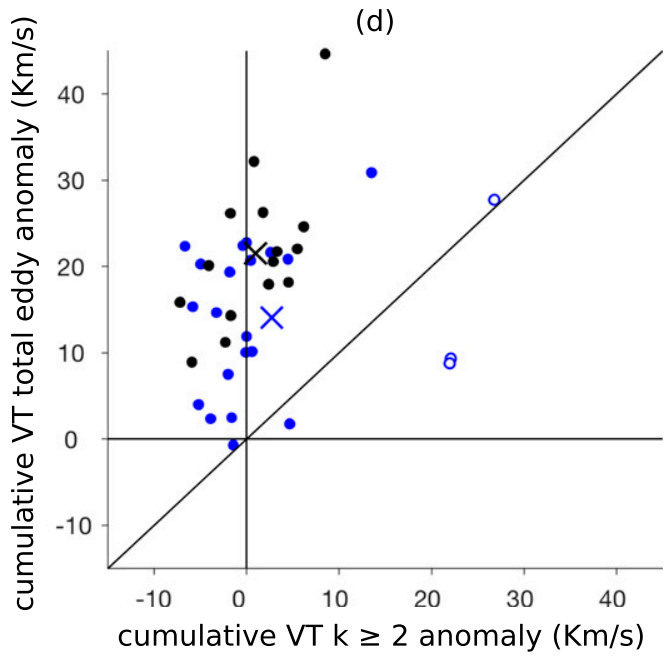

FIG. 1. (a) Anomalous wave- 1 vs eddy (all wavenumber) meridional heat flux averaged over $60^{\circ}-90^{\circ} \mathrm{N}$ at $50 \mathrm{hPa}$ over days -3 to +3 for individual extreme stratospheric eddy events in reanalysis (blue) and the model (black). Composite values are denoted with $\times$ symbols and the diagonal line denotes the one-to-one line. (b) As in (a), but for higher-order wavenumber $(k \geq 2)$ and eddy heat flux during extreme stratospheric eddy events. (c),(d) As in (a) and (b), respectively, but for the cumulative heat flux averaged 30 days prior to SSW events. Unfilled blue circles denote the split SSW events of January 1985, February 1989, and January 2009 in reanalysis data, which are dominated by the higher-order wavenumber component.

the anomalous tropospheric wave-1 heat flux prior to reanalysis events consistent with an anomalous tropospheric wave source.

Prior to extreme stratospheric eddy events in reanalysis, the stratospheric polar vortex is strengthened from days -20 to -10 , which is qualitatively captured by the model (Figs. 4a,b). In contrast, SSW events are preceded by a marginally strengthened polar vortex in the upper-stratosphere in both reanalysis and the model (Figs. 4c,d). We note that the stratospheric zonal-mean flow during SSW events is much weaker than extreme stratospheric eddy events (cf. Figs. 4a,b and c,d), showing that SSW events represent extreme zonal-mean flow events whereas extreme stratospheric eddy events represent zonal-mean flow deceleration events. Overall, the model qualitatively captures the strengthened vortex prior to extreme stratospheric eddy events in reanalysis consistent with a preconditioned stratospheric zonalmean flow. However, no clear preconditioning is found prior to SSW events in reanalysis or the model.

In summary, the results show the model captures the anomalous stratospheric wave-1 heat flux during 


\section{extreme stratospheric eddy}

(a)

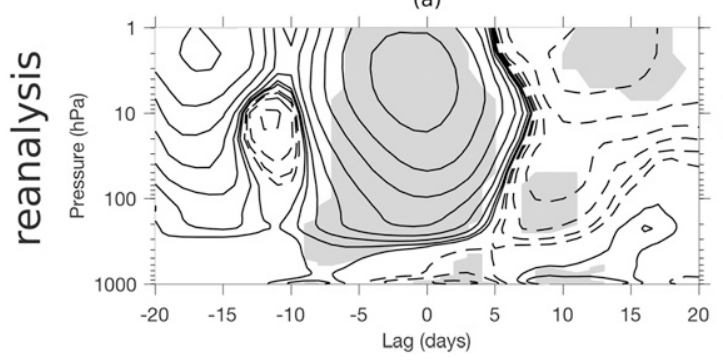

(b)

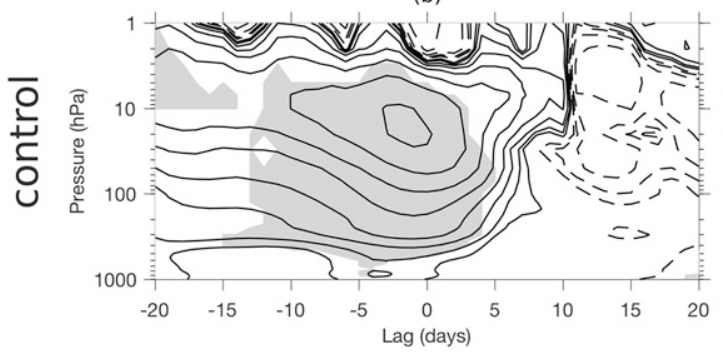

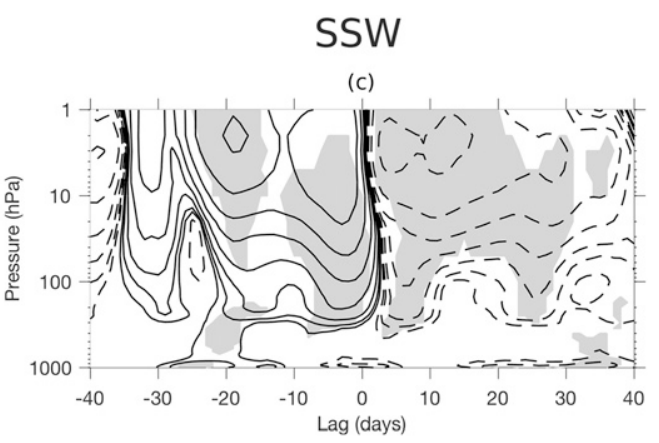

(d)

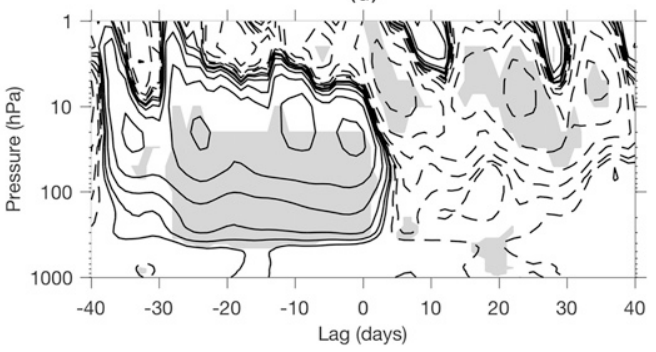

FIG. 2. Anomalous wave- 1 meridional heat flux averaged $60^{\circ}-90^{\circ} \mathrm{N}$ during composite (a),(b) extreme stratospheric eddy and (c),(d) SSW events in (a),(c) reanalysis and (b),(d) the model. Black contours are $\pm[1,2,4,8,16$, $32, \ldots] \mathrm{K} \mathrm{m} \mathrm{s}^{-1}$ and dashed contours are negative. Shading indicates statistical significance at the $95 \%$ level based on a two-tailed Student's $t$ test. Note the different length of time on the $x$-axis for extreme stratospheric eddy (days -20 to 20 ) vs SSW events (days -40 to 40 ).

extreme stratospheric eddy and SSW events in reanalysis data. The model qualitatively captures the anomalous tropospheric wave-1 heat flux preceding reanalysis events consistent with an anomalous source in the troposphere dominates view. However, in reanalysis and the model, only extreme stratospheric eddy events exhibit a strengthened vortex consistent with a preconditioned stratospheric zonal-mean flow in the stratosphere dominates view. Next, we use the nudging experiments to test the underlying mechanisms.

\section{b. Testing the troposphere and stratosphere dominates views}

If the troposphere dominates view is correct, then nudging tropospheric wave 1 alone should account for the anomalous stratospheric wave- 1 heat flux whereas nudging the stratospheric zonal-mean flow alone should not. Nudging tropospheric wave 1 (purple box, Figs. 5a,b) produces a positive stratospheric heat flux anomaly during extreme stratospheric eddy events (experiment 1 ) and prior to SSW events (experiment 2), accounting for 59\% of the instantaneous (days -3 to +3 ) and $94 \%$ of the cumulative (30 day) midstratospheric heat flux anomaly during the composite control events (blue line, Figs. 5a,b). In contrast, nudging the stratospheric zonalmean flow (red box in Figs. 5c,d) produces no stratospheric heat flux anomaly during extreme stratospheric eddy events (experiment 3 ) and a negative stratospheric heat flux anomaly prior to SSW events (experiment 4), accounting for $0 \%$ and $-55 \%$ of the composite control events in the midstratosphere (blue line in Figs. 5c,d).

We note that similar results are found when nudging the stratospheric zonal-mean flow only prior to extreme stratospheric eddy and SSW events (days -20 to -10 and days -40 to -20 ; Figs. S1a,b in the online supplemental material) or when reducing the nudging time scale by a factor of $10(\tau=1$ day; Figs. S1c,d), allowing for greater wave-mean flow interaction, which is key for the stratosphere dominates view (e.g., Holton and Mass 1976; Plumb 1981; Birner and Albers 2017). Qualitatively similar results are also found when repeating the experiments except for nudging above and below the Arctic tropopause defined at $298 \mathrm{hPa}$ (Fig. S2) and in a separate study nudging the stratospheric zonal-mean flow during SSWs (Hitchcock and Haynes 2016). Overall, the results are consistent with the troposphere dominates view: tropospheric wave 1 accounts for a large fraction of the anomalous stratospheric wave-1 heat flux whereas the stratospheric zonal-mean flow does not.

One of the caveats of the troposphere dominates view is that a small fraction of anomalous tropospheric eddy heat fluxes are followed by anomalous stratospheric eddy heat flux events, suggesting the stratospheric zonal-mean 
extreme stratospheric eddy

(a)

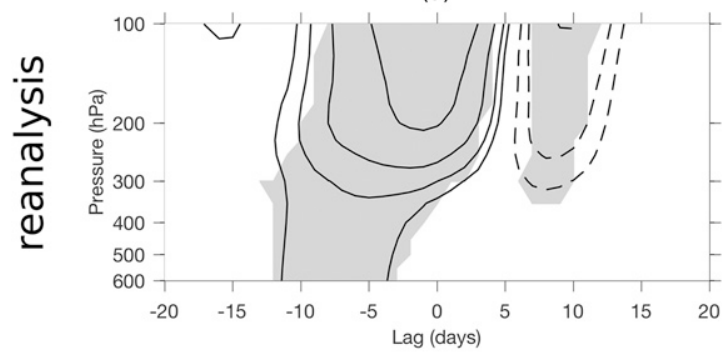

(b)

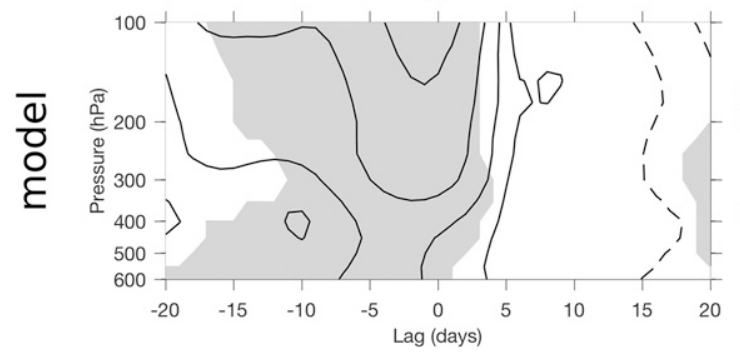

SSW

(c)

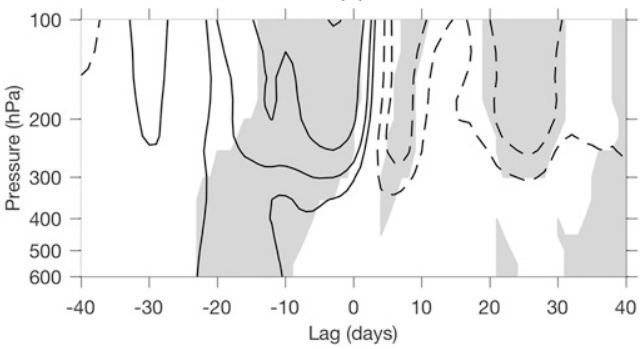

(d)

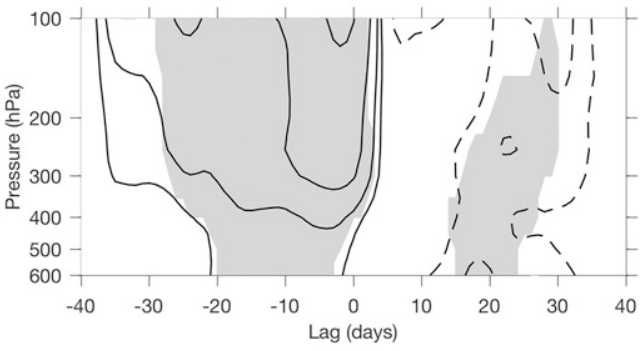

FIG. 3. Anomalous tropospheric wave- 1 meridional heat flux averaged over $40^{\circ}-70^{\circ} \mathrm{N}$ during composite (a),(b) extreme stratospheric eddy and (c),(d) SSW events in (a),(c) reanalysis and (b),(d) the model. Black contours are $\pm[1,2,4,8,16,32, \ldots] \mathrm{K} \mathrm{m} \mathrm{s}^{-1}$ and dashed contours are negative. Shading indicates statistical significance at the $95 \%$ level based on a two-tailed Student's $t$ test. The events are shown above the surface topography in the model, which extends to $600 \mathrm{hPa}$ in midlatitudes.

flow amplifies the stratospheric eddy heat flux (Birner and Albers 2017; de la Cámara et al. 2019). To better reconcile our results with the stratosphere dominates view, next we nudge tropospheric wave 1 in combination with the stratospheric zonal-mean flow. Nudging tropospheric wave 1 and the stratospheric zonal-mean flow in combination accounts for $89 \%$ and $74 \%$ of the composite control event heat flux in the midstratosphere during extreme stratospheric eddy (experiment 5) and prior to SSW events (experiment 6), respectively (blue line, Figs. 6a,b). The responses are amplified and damped in comparison to nudging tropospheric wave 1 alone (cf. Figs. 6a,b and Figs. 5a,b); however, SSWs are sensitive to the nudging tropopause level (cf. Figs. S3b and S2b). The results suggest the stratospheric zonal-mean flow can amplify the stratospheric heat flux from the troposphere.

If a specific configuration of the stratospheric zonalmean flow (Figs. 4b,d) is required to amplify the stratospheric eddy heat flux as hypothesized by the stratosphere dominates view, then nudging toward the climatological stratospheric zonal-mean flow should not amplify the stratospheric eddy heat flux. Nudging tropospheric wave 1 while also nudging the stratospheric zonal-mean flow to its climatology amplifies the stratospheric heat flux in comparison to nudging tropospheric wave 1 alone during extreme stratospheric eddy (experiment 7) and SSW events (experiment 8), respectively (cf. Figs. 6c,d and Figs. 5a,b).
Qualitatively similar results are found for nudging above and below the Arctic tropopause defined at $298 \mathrm{hPa}$ (cf. Figs. S3c,d and S2a,b). We confirm that nudging the climatological stratospheric zonal-mean flow alone produces a negligible wave- 1 heat flux response in the lower-stratosphere. Overall, the results suggest the climatological stratospheric zonal-mean flow is sufficient to account for the anomalous stratospheric heat flux generated in the troposphere consistent with the troposphere dominates view.

Finally, we examine whether tropospheric wave 1 or the stratospheric zonal-mean flow account for the anomalous zonal-mean flow in the stratosphere and troposphere. We focus on SSWs because they are associated with the largest zonal-mean flow anomalies. Beginning with the anomalous stratospheric zonalmean flow, nudging tropospheric wave 1 produces a large negative stratospheric zonal-mean flow anomaly but does not reverse the zonal-mean zonal wind at $60^{\circ} \mathrm{N}$ at $10 \mathrm{hPa}$ (cf. Fig. 7a and Fig. 4d). In contrast, nudging the stratospheric zonal-mean flow only prior to the events produces a weak response (days -40 to -2 ; Fig. S4a). Thus, tropospheric wave 1 does not capture the composite SSW but accounts for a large fraction of the anomalous stratospheric zonal-mean flow. A qualitatively similar but weaker response is found when nudging wave 1 below the Arctic tropopause at $298 \mathrm{hPa}$ 


\section{extreme stratospheric eddy}

(a)

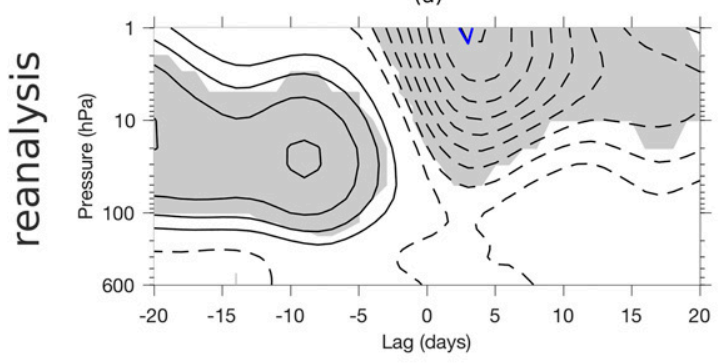

(b)

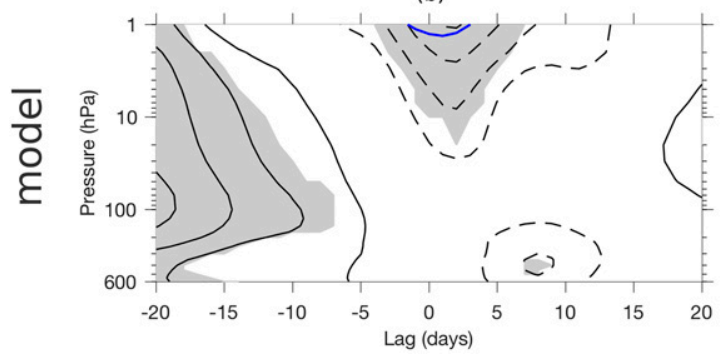

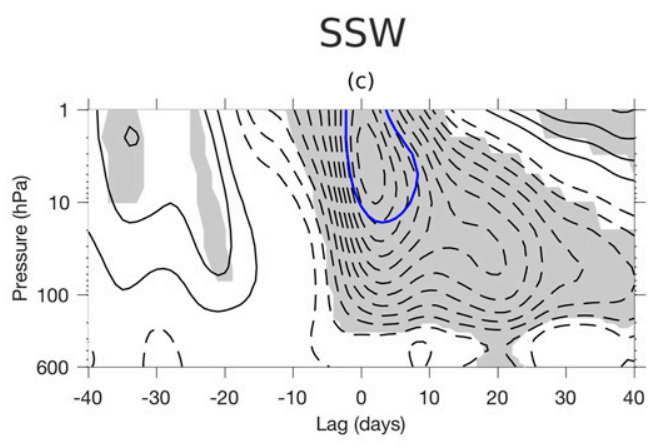

(d)

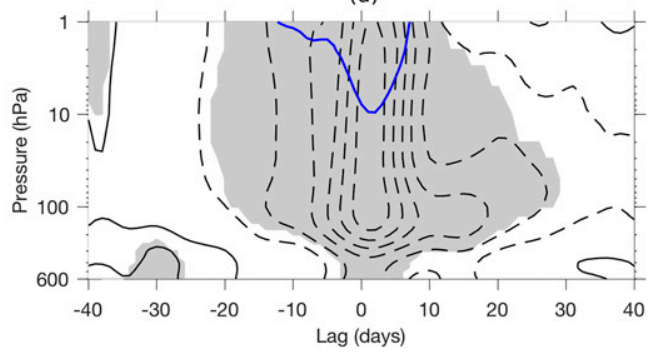

FIG. 4. NAM during composite (a),(b) extreme stratospheric eddy and (c),(d) SSW events in (a),(c) reanalysis and (b),(d) the model. Black contour interval is $0.25 \sigma$, dashed contours are negative, and the blue contour denotes where the total (anomaly plus climatology) zonal-mean zonal wind at $60^{\circ} \mathrm{N}$ is negative. The events are shown above the surface topography in the model, which extends to $600 \mathrm{hPa}$ in midlatitudes.

(Fig. S4b) consistent with previous work suggesting the upper troposphere-lower stratosphere region is important to drive SSW events (Birner and Albers 2017; de la Cámara et al. 2017, 2019).

Next, we consider the anomalous tropospheric zonal-mean flow. Nudging tropospheric wave 1 alone produces a negative tropospheric zonal-mean flow anomaly resembling the composite SSW (cf. Figs. 7a and $4 d$ ) suggesting it acts directly via the troposphere or indirectly via the anomalous stratospheric zonalmean flow. However, nudging tropospheric wave 1 while also nudging the stratospheric zonal-mean flow to its climatology produces a positive tropospheric zonalmean flow anomaly (Fig. 7d) whereas only experiments with an anomalous stratospheric zonal-mean flow involve a negative tropospheric zonal-mean flow anomaly (Figs. $7 \mathrm{a}-\mathrm{c}$ ). Qualitatively similar results are found when nudging above and below the Arctic tropopause (Figs. S4b-e). Thus, the anomalous stratospheric zonal-mean flow, independent of whether it reverses the zonal-mean zonal wind $60^{\circ} \mathrm{N}$ at $10 \mathrm{hPa}$, accounts for the anomalous tropospheric zonal-mean flow.

In summary, the results support the troposphere dominates view of the stratospheric wave- 1 heat flux during extreme stratospheric eddy and SSW events. Specifically, nudging tropospheric wave 1 alone or in combination with the stratospheric zonal-mean flow fixed to climatology accounts for a large fraction of the anomalous stratospheric wave- 1 heat flux. The results do not support the stratosphere dominates view since nudging the stratospheric zonal-mean flow alone does not account for the anomalous stratospheric wave- 1 heat flux. In contrast with the stratospheric heat flux during SSWs, tropospheric wave 1 does not capture the reversal of the zonal-mean zonal wind at $60^{\circ} \mathrm{N}$ at $10 \mathrm{hPa}$ but accounts for a large fraction of the anomalous stratospheric zonal-mean flow while the anomalous stratospheric zonal-mean flow accounts for the anomalous tropospheric zonal-mean flow.

\section{c. Quantifying the role of wave-wave interaction}

The results thus far suggest an anomalous tropospheric wave-1 source causes the anomalous stratospheric wave- 1 heat flux during extreme stratospheric eddy heat flux and SSW events. However, the troposphere dominates view discussed in the introduction does not specify what generates the anomalous wave- 1 source. If wave-wave interaction generates the tropospheric wave source, then nudging the tropospheric higher-order wavenumbers $(k \geq 2$, experiments 9 and 10) should account for the anomalous stratospheric and tropospheric wave-1 heat flux while nudging the stratospheric higherorder wavenumbers (experiments 11 and 12) should not.

The green colored boxes in Figs. 8a-d and $9 \mathrm{a}-\mathrm{d}$ denote the lag-pressure domains where the higher-order wavenumbers are nudged. Nudging the tropospheric higher- 


\section{extreme stratospheric eddy}

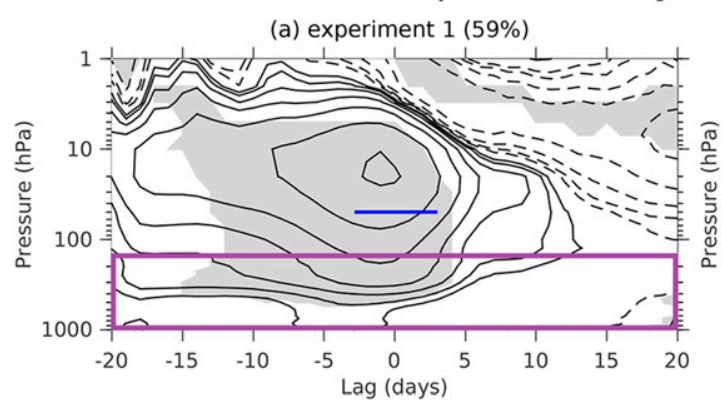

(c) experiment $3(0 \%)$

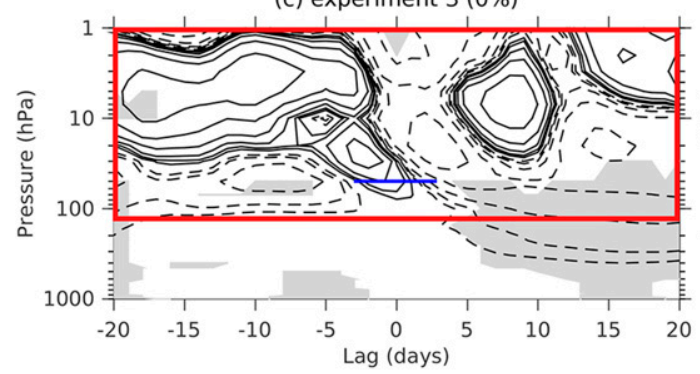

SSW

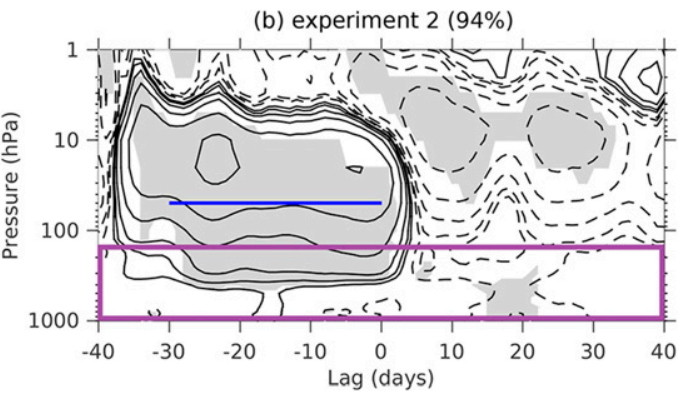

(d) experiment $4(-55 \%)$

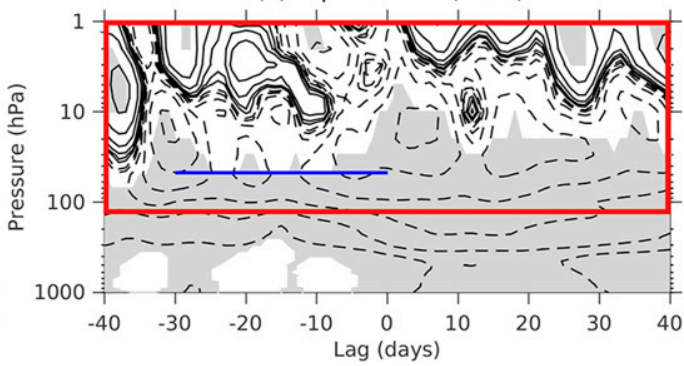

FIG. 5. As in Figs. 2b and 2d, but for the high-latitude wave-1 heat flux responses from experiments 1-4. The red and purple boxes denote the lag-pressure domains where the zonal-mean flow and wavenumber $\mathrm{k} 1$ are nudged, respectively. The values in parentheses quantify the percentage of the composite control event reproduced by each experiment denoted by the blue line.

order wavenumbers produces anomalous stratospheric wave-1 heat flux during extreme stratospheric eddy events and prior to SSW events, accounting for $41 \%$ of the instantaneous (days -3 to +3 ) and $51 \%$ of the cumulative (30 day) midstratospheric heat flux during the composite control events (blue line in Figs. 8a,b). Consistently, a large fraction of the tropospheric wave-1 heat flux anomaly prior to the events is reproduced $(65 \%$ and $114 \%$, Figs. 9a,b). In contrast, nudging the stratospheric higher-order wavenumbers produces weak wave-1 heat flux anomalies in the stratosphere $(14 \%$ and $-26 \%$, Figs. $8 \mathrm{c}, \mathrm{d})$ and troposphere $(13 \%$ and $15 \%$, Figs. $9 \mathrm{c}, \mathrm{d})$. The key role of the troposphere is confirmed by nudging the higherorder wavenumbers throughout the depth of the atmosphere but fixing tropospheric wave 1 to the time-independent climatology, resulting in weak stratospheric wave-1 heat flux anomalies (Fig. S5). Finally, we note that nudging the higher-order wavenumbers $(k \geq 2)$, which represent a large fraction of the tropospheric circulation, may artificially constrain the wave- 1 component. However, experiment A1 suggests that the circulation driven in our experiment is primarily a result of the event dynamics rather than the methodology (Fig. A1). Thus, the results suggest that tropospheric wave-wave interactions play a role in generating the anomalous tropospheric wave source during the events.
Wave-wave interaction could generate the anomalous tropospheric wave source directly via interaction between eddies (Scinocca and Haynes 1998; Schneidereit et al. 2017; Lee et al. 2019) or indirectly via the zonalmean flow (Charney and Eliassen 1949). If higher-order wavenumbers directly generate the anomalous tropospheric wave source, then fixing the zonal-mean flow to its time-independent climatology should still account for some fraction of the anomalous stratospheric and tropospheric wave-1 heat flux. The red and green colored boxes in Figs. 8e, 8f, 9e, and 9f denote the lag-pressure domains where the tropospheric zonal-mean flow is fixed and the higher-order wavenumbers are nudged. Nudging the tropospheric higher-order wavenumbers while fixing the tropospheric zonal-mean flow to climatology accounts for $42 \%$ and $63 \%$ of the composite control event heat flux in the midstratosphere during extreme stratospheric eddy (experiment 13) and prior to SSW events (experiment 14), respectively (blue line in Figs. 8e,f). Consistently, a large fraction of the positive tropospheric wave- 1 heat fluxes are also reproduced prior to both events (41\% and $163 \%$, Figs. 9e,f). We confirm the responses are due to nudging the tropospheric higher-order wavenumbers since fixing the tropospheric zonal-mean flow to climatology alone produces a negligible heat flux response in the troposphere and stratosphere. Thus, the results suggest that direct wave-wave interaction 
extreme stratospheric eddy

(a) experiment $5(89 \%)$

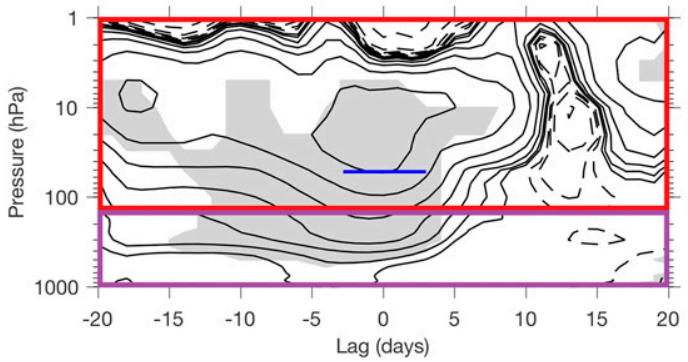

(c) experiment 7 (94\%)

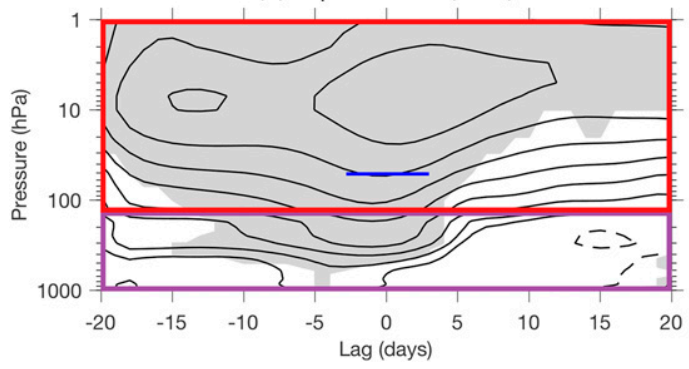

SSW

(b) experiment $6(72 \%)$

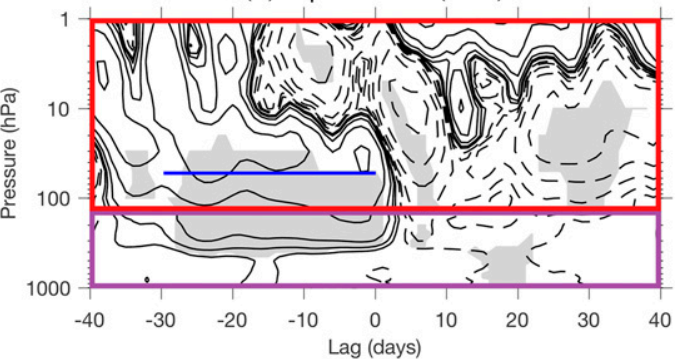

(d) experiment 8 (154\%)

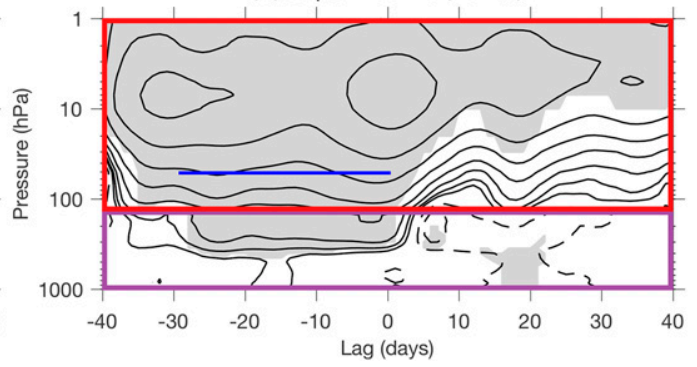

FIG. 6. As in Figs. 2b and 2d, but for the high-latitude wave-1 heat flux responses from experiments 5-8. The red and purple boxes denote the lag-pressure domains where the zonal-mean flow and wavenumber $\mathrm{k} 1$ are nudged, respectively. The values in parentheses quantify the percentage of the composite control event reproduced by each experiment denoted by the blue line.

plays a role in generating the anomalous tropospheric wave source.

In summary, the results suggest that tropospheric wave-wave interaction plays a role in generating the anomalous wave- 1 source during extreme stratospheric eddy heat flux and SSW events since nudging the higher-order wavenumbers in the troposphere accounts for a large fraction of the anomalous stratospheric and tropospheric wave-1 heat flux. The results provide a mechanism for generating the tropospheric wave source in the troposphere dominates view. Furthermore, the results suggest stratospheric wave-wave interactions do not play a role since nudging the stratospheric higherorder wavenumbers does not account for the stratospheric wave-1 heat flux.

\section{Conclusions and discussion}

\section{a. Conclusions}

Here we investigate whether the stratosphere or troposphere dominates the dynamics of the anomalous stratospheric eddy heat flux during extreme stratospheric eddy and SSW events. Ensemble spectral nudging experiments in a dry dynamical-core model were used to quantify the role of the troposphere versus the stratosphere. The experiments focused on the wavenumber- 1 heat flux since it dominates the anomalous eddy heat flux during the events (Fig. 1). Our results suggest the following answers to the questions posed in the introduction:

1) Does the troposphere or stratosphere dominate the dynamics of extreme stratospheric eddy and SSW events?

The results suggest tropospheric dynamics dominate the anomalous stratospheric wave- 1 heat flux; that is, the events involve an anomalous tropospheric source of wave activity propagating upward into the stratosphere (e.g., Matsuno 1971), since nudging tropospheric wave 1 alone or in combination with the stratospheric zonalmean flow fixed to climatology accounts for a large fraction of the anomalous stratospheric wave- 1 heat flux (Figs. 5a,b and 6c,d). The results do not support the view that stratospheric dynamics dominate (e.g., Holton and Mass 1976) since nudging the stratospheric zonal-mean flow alone does not account for the anomalous stratospheric wave-1 heat flux (Figs. 5c,d).

The results suggest both the troposphere and stratosphere dominate the dynamics of the zonalmean flow during SSWs. Tropospheric wave 1 does not capture the reversal of the zonal-mean zonal wind at $60^{\circ} \mathrm{N}$ at $10 \mathrm{hPa}$ but accounts for a large fraction of the anomalous stratospheric zonal-mean flow (Fig. 7a) while the anomalous stratospheric 


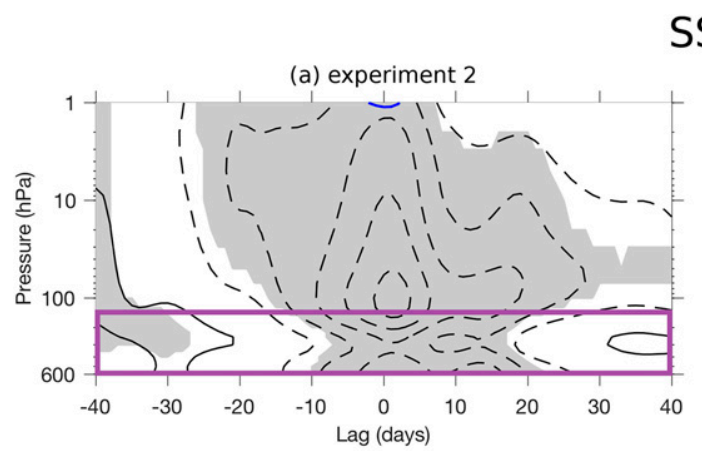

(c) experiment 6

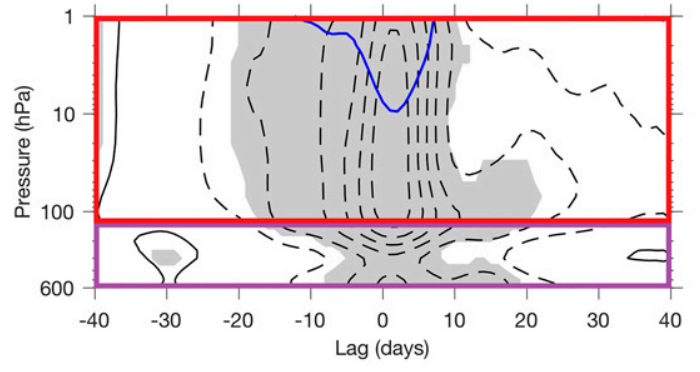

SSW

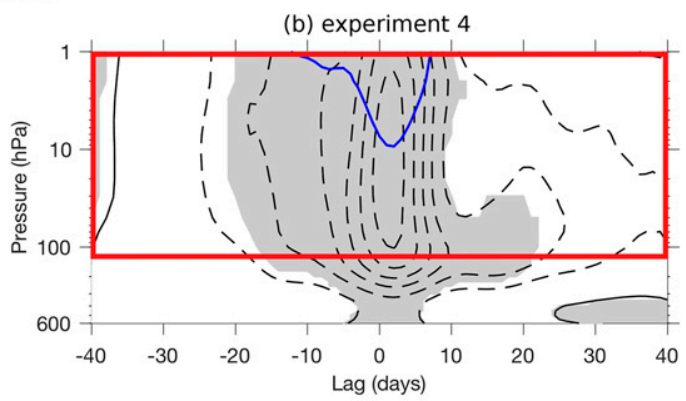

(d) experiment 8

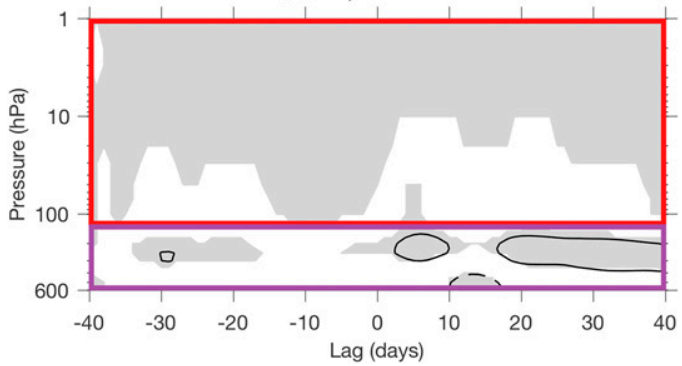

FIG. 7. As in Fig. 4d, but for NAM responses from experiments 2, 4, 6, and 8 . The red and purple boxes denote the lag-pressure domains where the zonal-mean flow and wavenumber k1 are nudged, respectively.

zonal-mean flow accounts for the anomalous tropospheric zonal-mean flow (Figs. 7a-c).

2) Do wave-wave interactions play a role during extreme stratospheric eddy and SSW events?

The results suggest tropospheric wave-wave interaction (Scinocca and Haynes 1998; Schneidereit et al. 2017; Lee et al. 2019) plays a role in generating the anomalous tropospheric wave- 1 source during extreme stratospheric eddy and SSW events. Nudging the tropospheric higher-order wavenumbers $(k \geq 2)$ alone or in combination with the tropospheric zonalmean flow fixed to climatology accounts for a large fraction of the anomalous stratospheric and tropospheric wave-1 heat flux (Figs. 8a,b,e,f and 9a,b,e,f). Conversely, the results suggest stratospheric wavewave interaction (McIntyre 1982; Palmer and Hsu 1983; Smith 1983; Smith et al. 1984; Albers and Birner 2014) does not play a role since nudging the stratospheric higher-order wavenumbers does not account for either (Figs. 8c,d) and 9c,d).

\section{b. Discussion}

Several previous studies have attempted to address the relative importance of the stratosphere versus the troposphere in producing the anomalous stratospheric eddy heat flux during SSW events. However, the studies are limited by testing the role of either the stratosphere or troposphere only, using diagnostics to infer causality, or deducing the behavior of the real atmosphere from highly truncated models (e.g., Matsuno 1971; Birner and Albers 2017; Hitchcock and Haynes 2016; de la Cámara et al. 2017). In contrast, Sun et al. (2012) used a dry dynamical-core model to perturb stratospheric and tropospheric initial conditions preceding SSW events and found the troposphere is key to predict the events. Our results confirm and extend the work of Sun et al. (2012) by using ensemble spectral nudging experiments in a dry dynamical-core model to 1) directly show the causal role of the troposphere versus the stratosphere by nudging the components throughout the duration of the events and 2) explicitly quantify the role of the tropospheric wave source versus the stratospheric zonal-mean flow consistent with the different views of planetary wave coupling.

Previous studies showed that most SSWs in reanalysis, idealized and state-of-the-art chemistry-climate models are preceded by upward tropospheric EP fluxes that are anomalous but not extreme $(<95$ th percentile), suggesting the troposphere plays a minor role in producing the anomalous stratospheric EP flux (Jucker 2016; Birner and Albers 2017; Lindgren et al. 2018; White et al. 2019; de la Cámara et al. 2019). While it is difficult to compare our results with previous studies because we focused on wave 1 instead of the eddy component $(k \geq 1)$, events in our model and reanalysis are preceded by tropospheric wave-1 EP fluxes that are anomalous but not extreme and our experiments demonstrate the dominant role of the troposphere in 


\section{extreme stratospheric eddy}

(a) experiment 9 (41\%)

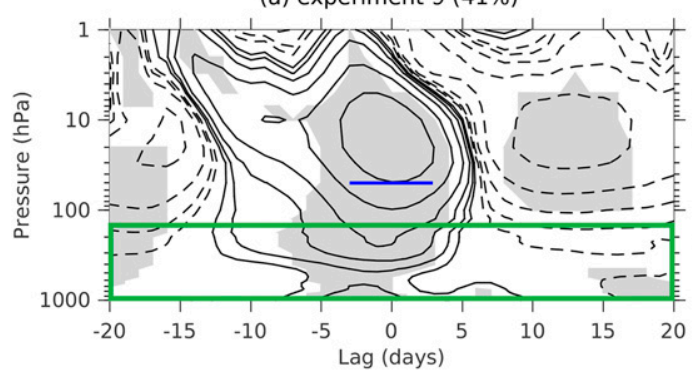

(c) experiment 11 (14\%)

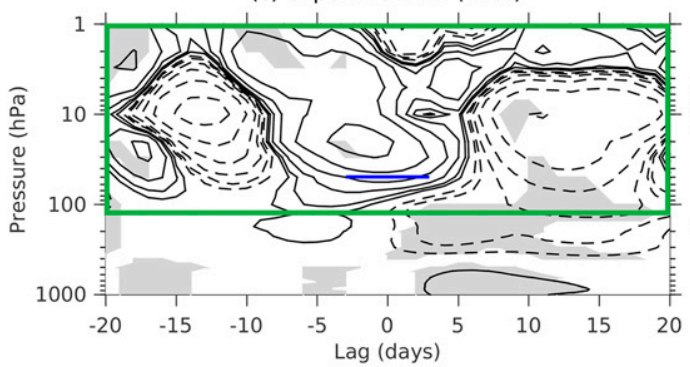

(e) experiment 13 (42\%)

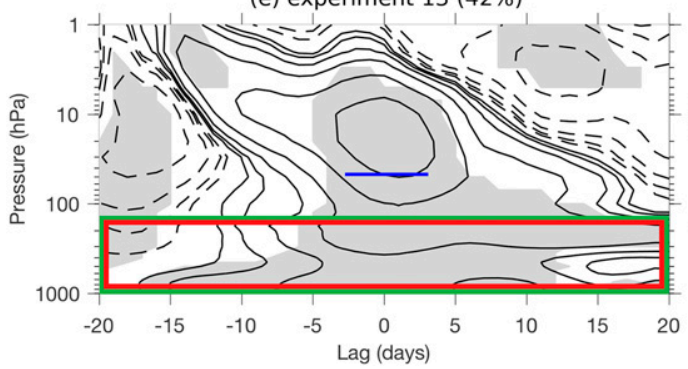

SSW

(b) experiment 10 (51\%)

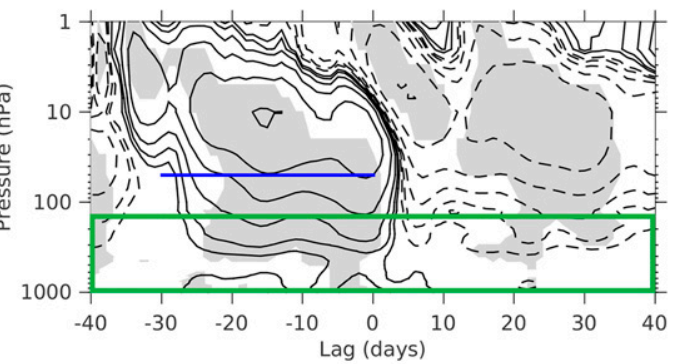

(d) experiment 12 (-26\%)

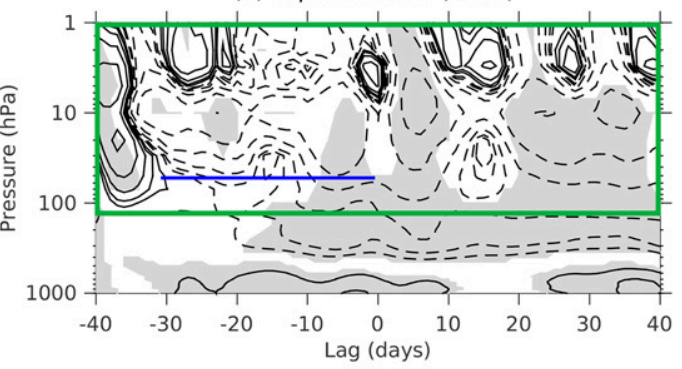

(f) experiment 14 (63\%)

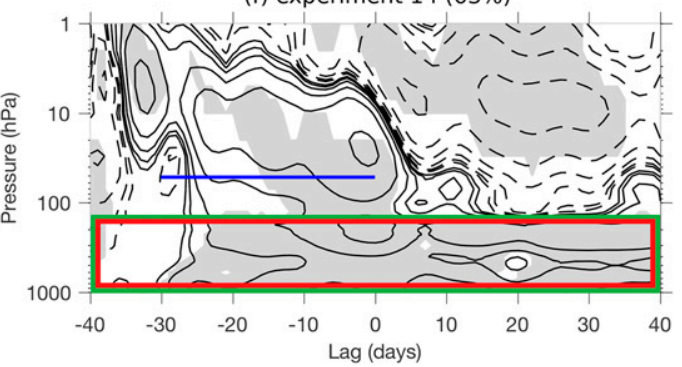

FIG. 8. As in Figs. 2b and 2d, but for the high-latitude wave-1 heat flux responses from experiments 9-14. The red and green boxes denote the lag-pressure domains where the zonal-mean flow and wavenumbers $k \geq 2$ are nudged, respectively. The values in parentheses quantify the percentage of the composite control event reproduced by each experiment denoted by the blue line.

producing the anomalous stratospheric wave-1 EP flux. We reason that because climatological $(\mathrm{k} 1$ or $k \geq 1)$ EP fluxes in the troposphere are larger magnitude than in the stratosphere, a small tropospheric EP-flux anomaly can produce a large stratospheric EP-flux anomaly for the same total (anomaly plus climatology) EP flux. Therefore, our results caution against using the anomalous tropospheric EP-flux diagnostic to infer causality.

Our results suggest both the troposphere and stratosphere are important to capture the zonal-mean flow evolution during SSWs consistent with previous studies. Specifically, the troposphere alone accounts for a large fraction of the anomalous stratospheric zonalmean flow but is not sufficient to capture the zonalmean zonal wind reversal at $60^{\circ} \mathrm{N}$ and $10 \mathrm{hPa}$ (de la Cámara et al. 2017) while the anomalous stratospheric zonal-mean flow accounts for the anomalous tropospheric zonal-mean flow (Hitchcock and Simpson 2014; Hitchcock and Haynes 2016).

The troposphere dominates view assumes an anomalous tropospheric source of stratospheric wave activity but does not specify how it is generated. Our results suggest nonlinear wave-wave interactions play role in generating the tropospheric wave source consistent with previous work using idealized and state-of-the-art models and reanalysis data (Scinocca and Haynes 1998; Schneidereit et al. 2017; Lee et al. 2019). However, we did not explicitly diagnose wavewave interactions in the model or reanalysis data and it is unclear why they play such an important role during extreme stratospheric eddy and SSW events in the model. Other processes in the real atmosphere that are absent in the idealized model could also play a role in generating the anomalous tropospheric wave source such as ENSO, the MJO, snow or sea ice (e.g., Domeisen et al. 2019; Henderson et al. 2018; Kang and Tziperman 2018; Screen et al. 2018). 


\section{extreme stratospheric eddy}

(a) experiment $9(65 \%)$

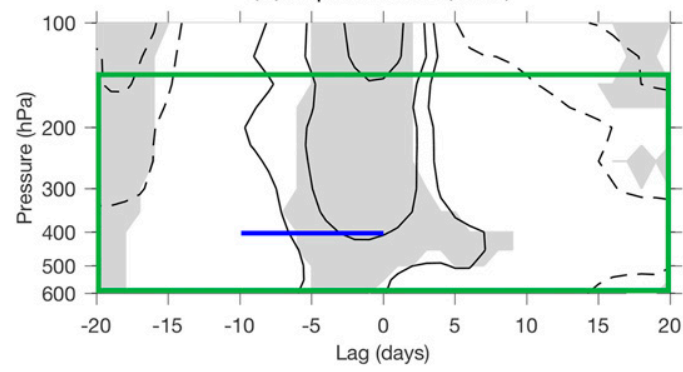

(c) experiment $11(13 \%)$

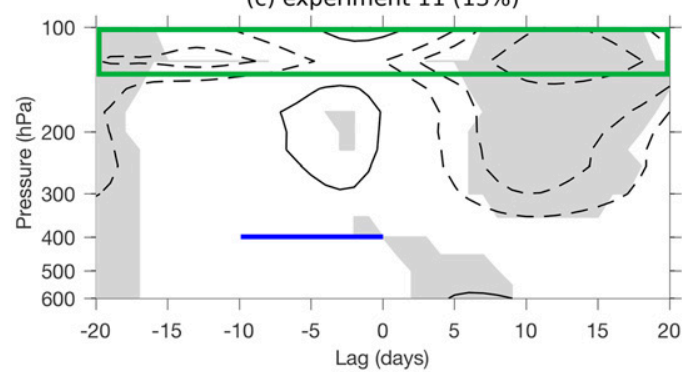

(e) experiment $13(41 \%)$

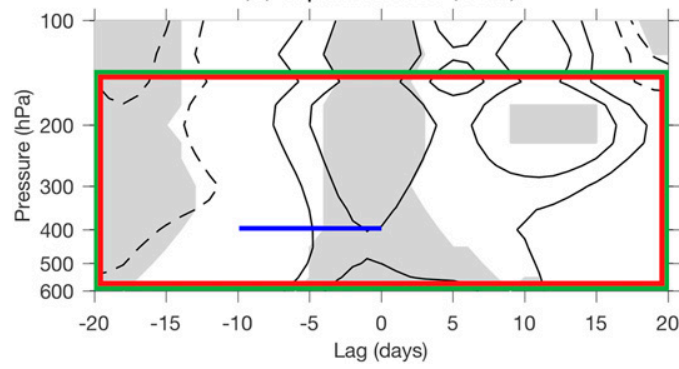

SSW

(b) experiment 10 (114\%)

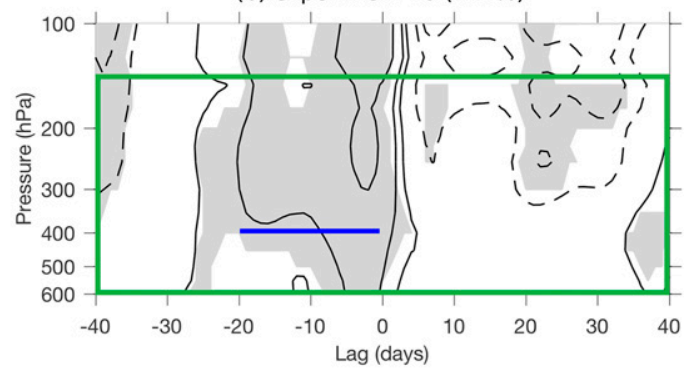

(d) experiment 12 (15\%)

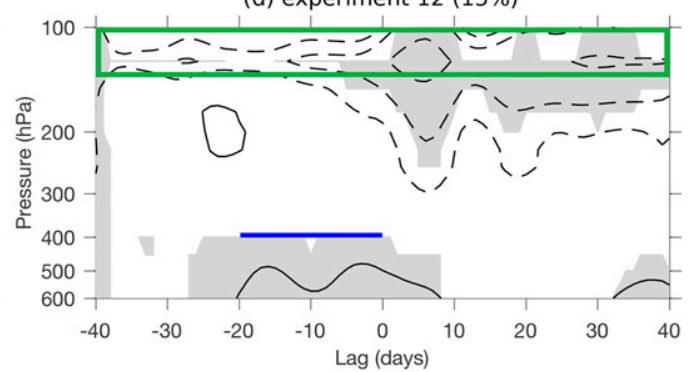

(f) experiment $14(163 \%)$

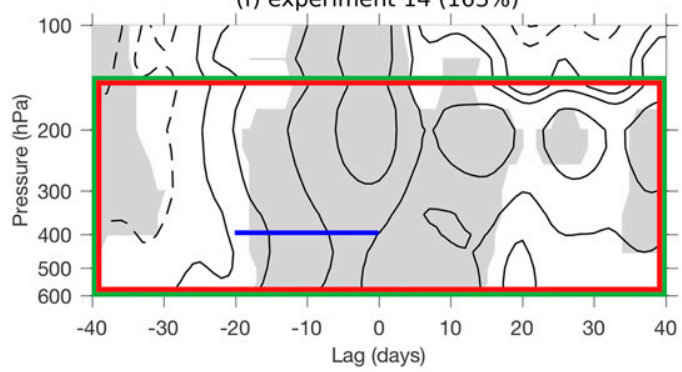

FIG. 9. As in Figs. 3b and 3d, but for the wave- 1 heat flux responses averaged over $40^{\circ}-70^{\circ} \mathrm{N}$ from experiments 9-14. The red and green boxes denote the lag-pressure domains where the zonal-mean flow and wavenumbers $k \geq 2$ are nudged, respectively. The values in parentheses quantify the percentage of the composite control event reproduced by each experiment denoted by the blue line.

Acknowledgments. E.D.S. thanks Camille Li, Paul Kushner, Isla Simpson, and Karen Smith for helpful discussions. E.D.S and T.A.S thank the three anonymous reviewers for their constructive comments.

Data availability statement. ERA-Interim data can be obtained at https://apps.ecmwf.int/datasets/data/ interim-full-daily/. All model data are available from E.D.S. upon request.

\section{APPENDIX}

\section{Sensitivity of Results to Nudging Higher-Order Wavenumbers}

The results suggest that tropospheric higher-order wavenumbers can account for a large fraction of the anomalous stratospheric wave- 1 heat flux during the events (Figs. 9a,b). However, the results rely on the assumption that the circulation driven by the nudging is a result of the event dynamics rather than the nudging itself. The assumption may not hold in a tightly coupled system when nudging higher-order wavenumbers $(k \geq 2)$, which represent a large fraction of the tropospheric circulation. Therefore, we test the sensitivity to the nudging methodology by nudging the tropospheric higher-order wavenumbers to the evolution of the control simulation from days 500 to 3500 in a single integration (experiment A1). If the results are an artifact of the nudging, then the tropospheric and stratospheric wave- 1 heat flux from the control simulation should be reproduced. Figure A1 shows the variance explained $R^{2}$ by correlating the tropospheric (black line, $40^{\circ}-70^{\circ} \mathrm{N}$ ) and stratospheric (blue line, $60^{\circ}-90^{\circ} \mathrm{N}$ ) 


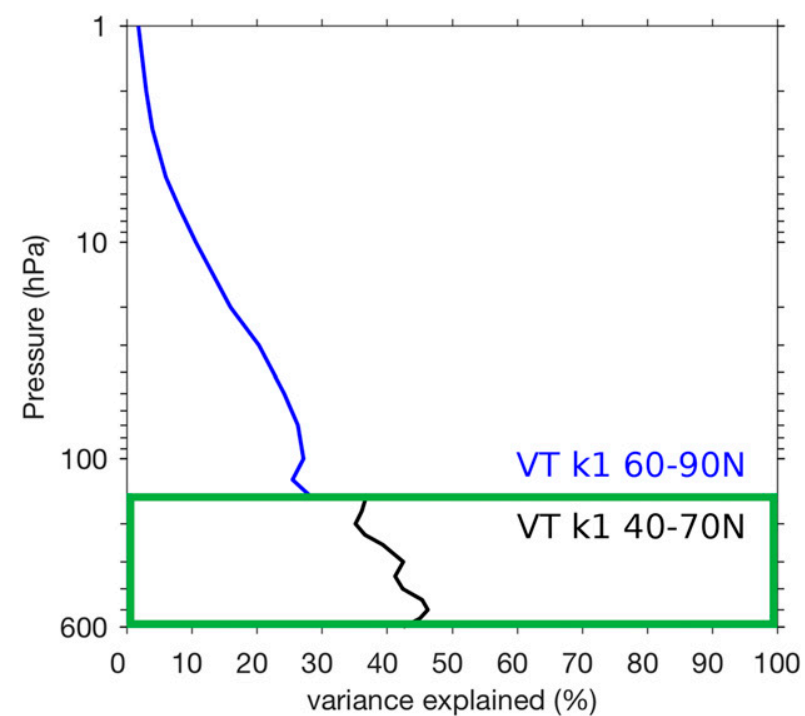

FIG. A1. Percent variance explained $R^{2}$ by correlating the daily tropospheric $\left(40^{\circ}-70^{\circ} \mathrm{N}\right.$; black $)$ and stratospheric $\left(60^{\circ}-90^{\circ} \mathrm{N}\right.$; blue $)$ wave-1 meridional heat flux from experiment $\mathrm{A} 1$ with the control simulation from days 500 to 3500 . Green box denotes the pressure domain where the higher-order wavenumbers $(k \geq 2)$ are nudged.

wave-1 heat flux from experiment A1 with the control simulation. The results show that nudging the tropospheric higher-order wavenumbers accounts for about $20 \%$ and $40 \%$ of the wave- 1 heat flux variance in the stratosphere and troposphere, respectively. The nudging poorly constrains the wave- 1 heat flux in the stratosphere and constrains less than half the variability in the troposphere. Thus, we conclude that the circulation driven by nudging the tropospheric higher-order wavenumbers is primarily a result of the event dynamics rather than the nudging itself.

\section{REFERENCES}

Albers, J. R., and T. Birner, 2014: Vortex preconditioning due to planetary and gravity waves prior to sudden stratospheric warmings. J. Atmos. Sci., 71, 4028-4054, https://doi.org/ 10.1175/JAS-D-14-0026.1.

Andrews, D. G., J. R. Holton, and C. B. Leovy, 1987: Middle Atmosphere Dynamics. Vol. 40. Academic Press, 489 pp.

Baldwin, M. P., and T. J. Dunkerton, 2001: Stratospheric harbingers of anomalous weather regimes. Science, 294, 581-584, https://doi.org/10.1126/science.1063315.

Birner, T., and J. R. Albers, 2017: Sudden stratospheric warmings and anomalous upward wave activity flux. SOLA, 13A, 8-12, https://doi.org/10.2151/SOLA.13A-002.

Butler, A. H., J. P. Sjoberg, D. J. Seidel, and K. H. Rosenlof, 2017: A sudden stratospheric warming compendium. Earth Syst. Sci. Data, 9, 63-76, https://doi.org/10.5194/essd-9-63-2017.

Charlton, A. J., and L. M. Polvani, 2007: A new look at stratospheric sudden warmings. Part I: Climatology and modeling benchmarks. J. Climate, 20, 449-469, https://doi.org/10.1175/JCLI3996.1.

Charney, J. G., and A. Eliassen, 1949: A numerical method for predicting the perturbations of the middle latitude westerlies. Tellus, 1 (2), 38-54, https://doi.org/10.3402/tellusa.v1i2.8500.
— , and P. G. Drazin, 1961: Propagation of planetary-scale disturbances from the lower into the upper atmosphere. J. Geophys. Res., 66, 83-109, https://doi.org/10.1029/JZ066i001p00083.

Chen, P., and W. A. Robinson, 1992: Propagation of planetary waves between the troposphere and stratosphere. J. Atmos. Sci., 49, 2533-2545, https://doi.org/10.1175/1520-0469(1992) 049<2533:POPWBT >2.0.CO;2.

Clark, J. H., 1974: Atmospheric response to the quasi-resonant growth of forced planetary waves. J. Meteor. Soc. Japan, 52, 143-163, https://doi.org/10.2151/jmsj1965.52.1_143.

Dee, D. P., and Coauthors, 2011: The ERA-Interim reanalysis: Configuration and performance of the data assimilation system. Quart. J. Roy. Meteor. Soc., 137, 553-597, https://doi.org/ 10.1002/qj.828.

de la Cámara, A., J. R. Albers, T. Birner, R. R. Garcia, P. Hitchcock, D. E. Kinnison, and A. K. Smith, 2017: Sensitivity of sudden stratospheric warmings to previous stratospheric conditions. J. Atmos. Sci., 74, 2857-2877, https://doi.org/10.1175/JAS-D-170136.1 .

, T. Birner, and J. R. Albers, 2019: Are sudden stratospheric warmings preceded by anomalous tropospheric wave activity? J. Climate, 32, 7173-7189, https://doi.org/10.1175/JCLI-D-190269.1 .

Domeisen, D. I., and R. A. Plumb, 2012: Traveling planetary-scale Rossby waves in the winter stratosphere: The role of tropospheric baroclinic instability. Geophys. Res. Lett., 39, L20817, https://doi.org/10.1029/2012GL053684.

_ - O. Martius, and B. Jiménez-Esteve, 2018: Rossby wave propagation into the Northern Hemisphere stratosphere: The role of zonal phase speed. Geophys. Res. Lett., 45, 2064-2071, https://doi.org/10.1002/2017GL076886.

— C. I. Garfinkel, and A. H. Butler, 2019: The teleconnection of El Niño Southern Oscillation to the stratosphere. Rev. Geophys., 57, 5-47, https://doi.org/10.1029/2018RG000596.

Douville, H., 2009: Stratospheric polar vortex influence on Northern Hemisphere winter climate variability. Geophys. Res. Lett., 36, L18703, https://doi.org/10.1029/2009GL039334.

Dunn-Sigouin, E., and T. A. Shaw, 2015: Comparing and contrasting extreme stratospheric events, including their coupling to the tropospheric circulation. J. Geophys. Res. Atmos., 120, 1374-1390, https://doi.org/10.1002/2014JD022116.

— and - 2018: Dynamics of extreme stratospheric negative heat flux events in an idealized model. J. Atmos. Sci., 75, 35213540, https://doi.org/10.1175/JAS-D-17-0263.1.

Esler, J., and N. J. Matthewman, 2011: Stratospheric sudden warmings as self-tuning resonances. Part II: Vortex displacement events. J. Atmos. Sci., 68, 2505-2523, https://doi.org/ 10.1175/JAS-D-11-08.1.

Gerber, E. P., and Coauthors, 2010: Stratosphere-troposphere coupling and annular mode variability in chemistry-climate models. J. Geophys. Res., 115, D00M06, https://doi.org/ 10.1029/2009JD013770.

Greatbatch, R. J., G. Gollan, T. Jung, and T. Kunz, 2012: Factors influencing Northern Hemisphere winter mean atmospheric circulation anomalies during the period 1960/61 to 2001/02. Quart. J. Roy. Meteor. Soc., 138, 1970-1982, https://doi.org/10.1002/qj.1947.

Harnik, N., 2009: Observed stratospheric downward reflection and its relation to upward pulses of wave activity. J. Geophys. Res., 114, D08120, https://doi.org/10.1029/2008JD010493.

Henderson, G. R., Y. Peings, J. C. Furtado, and P. J. Kushner, 2018: Snow-atmosphere coupling in the Northern Hemisphere. Nat. Climate Change, 8, 954-963, https://doi.org/10.1038/ S41558-018-0295-6. 
Hitchcock, P., and I. R. Simpson, 2014: The downward influence of stratospheric sudden warmings. J. Atmos. Sci., 71, 3856-3876, https://doi.org/10.1175/JAS-D-14-0012.1.

— waves. Geophys. Res. Lett., 43, 11 884-11 892, https://doi.org/ 10.1002/2016GL071372.

Holton, J. R., and C. Mass, 1976: Stratospheric vacillation cycles. J. Atmos. Sci., 33, 2218-2225, https://doi.org/10.1175/15200469(1976)033<2218:SVC>2.0.CO;2.

Jucker, M., 2016: Are sudden stratospheric warmings generic? Insights from an idealized GCM. J. Atmos. Sci., 73, 5061-5080, https://doi.org/10.1175/JAS-D-15-0353.1.

Kang, W., and E. Tziperman, 2018: The role of zonal asymmetry in the enhancement and suppression of sudden stratospheric warming variability by the Madden-Julian oscillation. J. Climate, 31, 23992415, https://doi.org/10.1175/JCLI-D-17-0489.1.

Karoly, D. J., and B. J. Hoskins, 1982: Three dimensional propagation of planetary waves. J. Meteor. Soc. Japan, 60, 109-123, https://doi.org/10.2151/jmsj1965.60.1_109.

Lee, S. H., A. J. Charlton-Perez, J. C. Furtado, and S. J. Woolnough, 2019: Abrupt stratospheric vortex weakening associated with North Atlantic anticyclonic wave breaking. J. Geophys. Res. Atmos., 124, 8563-8575, https://doi.org/ 10.1029/2019JD030940.

Limpasuvan, V., D. W. Thompson, and D. L. Hartmann, 2004: The life cycle of the Northern Hemisphere sudden stratospheric warmings. J. Climate, 17, 2584-2596, https://doi.org/10.1175/ 1520-0442(2004)017<2584:TLCOTN >2.0.CO;2.

Lindgren, E., A. Sheshadri, and R. Plumb, 2018: Sudden stratospheric warming formation in an idealized general circulation model using three types of tropospheric forcing. J. Geophys. Res. Atmos., 123, 10-125, https://doi.org/ 10.1029/2018JD028537.

Martineau, P., and S.-W. Son, 2015: Onset of circulation anomalies during stratospheric vortex weakening events: The role of planetary-scale waves. J. Climate, 28, 7347-7370, https://doi.org/ 10.1175/JCLI-D-14-00478.1.

—, G. Chen, S.-W. Son, and J. Kim, 2018: Lower-stratospheric control of the frequency of sudden stratospheric warming events. J. Geophys. Res. Atmos., 123, 3051-3070, https:// doi.org/10.1002/2017JD027648.

Matsuno, T., 1970: Vertical propagation of stationary planetary waves in the winter Northern Hemisphere. J. Atmos. Sci., 27, 871-883, https://doi.org/10.1175/1520-0469(1970)027<0871: VPOSPW $>2.0 . \mathrm{CO} ; 2$.

- 1971: A dynamical model of the stratospheric sudden warming. J. Atmos. Sci., 28, 1479-1494, https://doi.org/10.1175/ 1520-0469(1971)028<1479:ADMOTS > 2.0.CO;2.

Matthewman, N. J., and J. G. Esler, 2011: Stratospheric sudden warmings as self-tuning resonances. Part I: Vortex splitting events. J. Atmos. Sci., 68, 2481-2504, https://doi.org/10.1175/ JAS-D-11-07.1.

McIntyre, M. E., 1982: How well do we understand the dynamics of stratospheric warmings? J. Meteor. Soc. Japan, 60, 37-65, https://doi.org/10.2151/jmsj1965.60.1_37.

Palmer, T., and C. F. Hsu, 1983: Stratospheric sudden coolings and the role of nonlinear wave interactions in preconditioning the circumpolar flow. J. Atmos. Sci., 40, 909-928, https://doi.org/ 10.1175/1520-0469(1983)040<0909:SSCATR $>2.0 . C O ; 2$.

Plumb, R. A., 1981: Instability of the distorted polar night vortex: A theory of stratospheric warmings. J. Atmos. Sci., 38, 2514-2531, https://doi.org/10.1175/1520-0469(1981)038<2514: IOTDPN>2.0.CO;2.
_ 2010: Planetary waves and the extratropical winter stratosphere. The Stratosphere: Dynamics, Transport and Chemistry, Geophys. Monogr., Vol. 190, Amer. Geophys. Union, 23-42, https://doi.org/10.1029/2009GM000888.

Polvani, L. M., and D. W. Waugh, 2004: Upward wave activity flux as a precursor to extreme stratospheric events and subsequent anomalous surface weather regimes. J. Climate, 17, 3548-3554, https:// doi.org/10.1175/1520-0442(2004)017<3548:UWAFAA >2.0.CO;2.

Reichler, T., P. J. Kushner, and L. M. Polvani, 2005: The coupled stratosphere-troposphere response to impulsive forcing from the troposphere. J. Atmos. Sci., 62, 3337-3352, https://doi.org/ 10.1175/JAS3527.1.

Schneidereit, A., and Coauthors, 2017: Enhanced tropospheric wave forcing of two anticyclones in the prephase of the January 2009 major stratospheric sudden warming event. Mon. Wea. Rev., 145, 1797-1815, https://doi.org/10.1175/MWR-D-16-0242.1.

Scinocca, J. F., and P. H. Haynes, 1998: Dynamical forcing of stratospheric planetary waves by tropospheric baroclinic eddies. J. Atmos. Sci., 55, 2361-2392, https://doi.org/10.1175/ 1520-0469(1998)055<2361:DFOSPW >2.0.CO;2.

Scott, R., and L. M. Polvani, 2006: Internal variability of the winter stratosphere. Part I: Time-independent forcing. J. Atmos. Sci., 63, 2758-2776, https://doi.org/10.1175/JAS3797.1.

Screen, J. A., and Coauthors, 2018: Consistency and discrepancy in the atmospheric response to Arctic sea-ice loss across climate models. Nat. Geosci., 11, 155-163, https://doi.org/10.1038/ s41561-018-0059-y.

Shaw, T. A., and J. Perlwitz, 2013: The life cycle of Northern Hemisphere downward wave coupling between the stratosphere and troposphere. J. Climate, 26, 1745-1763, https:// doi.org/10.1175/JCLI-D-12-00251.1.

,-- , and N. Harnik, 2010: Downward wave coupling between the stratosphere and troposphere: The importance of meridional wave guiding and comparison with zonal-mean coupling. J. Climate, 23, 6365-6381, https://doi.org/10.1175/ 2010JCLI3804.1.

Sjoberg, J. P., and T. Birner, 2012: Transient tropospheric forcing of sudden stratospheric warmings. J. Atmos. Sci., 69, 34203432, https://doi.org/10.1175/JAS-D-11-0195.1.

$\longrightarrow$, and - , 2014: Stratospheric wave-mean flow feedbacks and sudden stratospheric warmings in a simple model forced by upward wave activity flux. J. Atmos. Sci., 71, 4055-4071, https://doi.org/10.1175/JAS-D-14-0113.1.

Smith, A. K., 1983: Observation of wave-wave interactions in the stratosphere. J. Atmos. Sci., 40, 2484-2496, https://doi.org/ 10.1175/1520-0469(1983)040<2484:OOWWII >2.0.CO;2.

_ J. J. Gille, and L. V. Lyjak, 1984: Wave-wave interactions in the stratosphere: Observations during quiet and active wintertime periods. J. Atmos. Sci., 41, 363-373, https://doi.org/ 10.1175/1520-0469(1984)041<0363:WIITSO > 2.0.CO;2.

Sun, L., W. A. Robinson, and G. Chen, 2012: The predictability of stratospheric warming events: More from the troposphere or the stratosphere? J. Atmos. Sci., 69, 768-783, https://doi.org/ 10.1175/JAS-D-11-0144.1.

Tung, K. K., and R. Lindzen, 1979: A theory of stationary long waves. Part I: A simple theory of blocking. Mon. Wea. Rev., 107, 714-734, https://doi.org/10.1175/1520-0493(1979)107<0714: ATOSLW $>2.0 . \mathrm{CO} ; 2$.

White, I., C. I. Garfinkel, E. P. Gerber, M. Jucker, V. Aquila, and L. D. Oman, 2019: The downward influence of sudden stratospheric warmings: Association with tropospheric precursors. J. Climate, 32, 85-108, https://doi.org/10.1175/JCLID-18-0053.1. 